\title{
CSF rhinorrhea complicated by recurrent pyogenic meningitis due to congenital anterior skull base defect: Report of two cases
}

\author{
Radheshyam Purkait, Biswajit Das, Suman Bhattacharya, Ashis Saha, Sudipta \\ Kar, Birendranath Roy \\ (Department of Pediatric Medicine, NilRatan Sircar Medical College and Hospital, India)
}

\begin{abstract}
Cerebrospinal fluid (CSF) rhinorrhea is the result of an osseous defect at the skull base coupled with a disruption of the dura mater and arachnoid with a resultant pressure gradient leading to the CSF leak. Cerebrospinal fluid (CSF) rhinorrhea is a potentially dangerous condition as the risk of intracranial infection is increased if it persists. Therefore, accurate preoperative localization of the site of leakage as well as its appropriate management is mandatory. We here report two children with CSF rhinorrhea due to congenital anterior skull base defect, complicated by recurrent pyogenic meningitis, underwent surgical repair with uneventful post-operative period. In this article, etiologies, laboratory investigation including imaging and management are also discussed in brief.
\end{abstract}

Keywords: Cerebrospinal fluid (CSF) rhinorrhea, Pyogenic meningitis, Meningoencephalocele

\section{INTRODUCTION}

Cerebrospinal fluid (CSF) rhinorrhea is the leakage of CSF from subarachnoid space into the nasal cavity due to defect in the dura matter, bone and mucosa. The fluid may escape directly into the nose from a defect in the anterior cranial fossa either via the frontal, ethmoid or sphenoid sinuses or from the cribriform plate [1]. Recent study indicates that there is a 10\% annual risk of developing meningitis in patients with CSF leaks if left untreated [2]. We report two children with CSF rhinorrhea due to congenital anterior skull base defect, complicated by recurrent ascending pyogenic meningitis, underwent surgical repair with uneventful postoperative period. This article also reviewed in brief the causes, diagnosis and treatment of CSF rhinorrhea.

1. Case 1

\section{HEADINGS}

1.1 Case History: A 5-year-old boy presented with complaints of clear fluid leaking from left side of nose off and on since last 8 months. The discharge accentuated on bending forward, coughing, sneezing and more profuse when arising from bed and changing positions particularly tilting head to the left side. Child had to be admitted twice for high grade fever associated with headache during this period and subsequently diagnosed as bacterial meningitis. He denied any history of head trauma or intracranial surgery.

1.2 Investigations: The glucose content of the collected nasal secretion was $40 \mathrm{mg} / \mathrm{dL}$ and biochemical analysis for beta-2 transferrin was positive, consistent with CSF. A computed tomography (CT) cisternography was performed to locate the site of leakage. It showed a bony defect in the left anterior cribriform plate through which a mass with mixed soft tissue and fluid attenuation was seen herniating into the left ethmoid sinuses and nasal cavity [Fig. 1a]. Magnetic resonance imaging (MRI) suggested the diagnosis of transethmoidal meningoencephalocele [Fig. 1b\&c].

1.3 Treatment: The child underwent surgical repair by extracranial approach via lateral rhinotomy. The defect in the cribriform plate was accessed and the protruding brain tissue was cauterized and removed. The bony gap was patched with graft harvested from the fascia lata, also supported by nasal mucosal flap. Following surgery, no further recurrence of symptoms was observed till date. The histological examination of the biopsy materials revealed the presence of brain tissue and fragments of fibrocollagenous tissue covered by both respiratory epithelium and meningothelial cells compatible with meningoencephalocele.

\section{Case 2}

2.1 Case History: An 8-year-old boy presented with recurrent pyogenic meningitis and intermittent rhinorrhea comprising clear watery fluid from the left nostril since last 18 months. It was every time preceded by mild headache that used to relieve when the leak occurs. He could not recall any history of head trauma or surgery. 2.2 Investigations: After the biochemical conformation of the nasal secretion as CSF, high resolution CT (HRCT) scan was done which showed a bony gap $(1 \mathrm{~mm})$ in the medial part of left cribriform plate but no CSF leak was demonstrated [Fig. 2]. MRI of brain failed to detect any CSF fistula. 
2.3 Treatment: In the view of recurrent meningitis, child was planned for surgical repair by endoscopic approach. The exact location of CSF leak was detected intra-operatively and the mucosa surrounding the defect was excised. Perichondrial graft material was placed into the defect as underlay graft and then sealed with fibrin glue. The patient was under follow-up for last ten month and no recurrence of CSF leak or meningitis is reported till date.

III. FIGURES AND TABLES

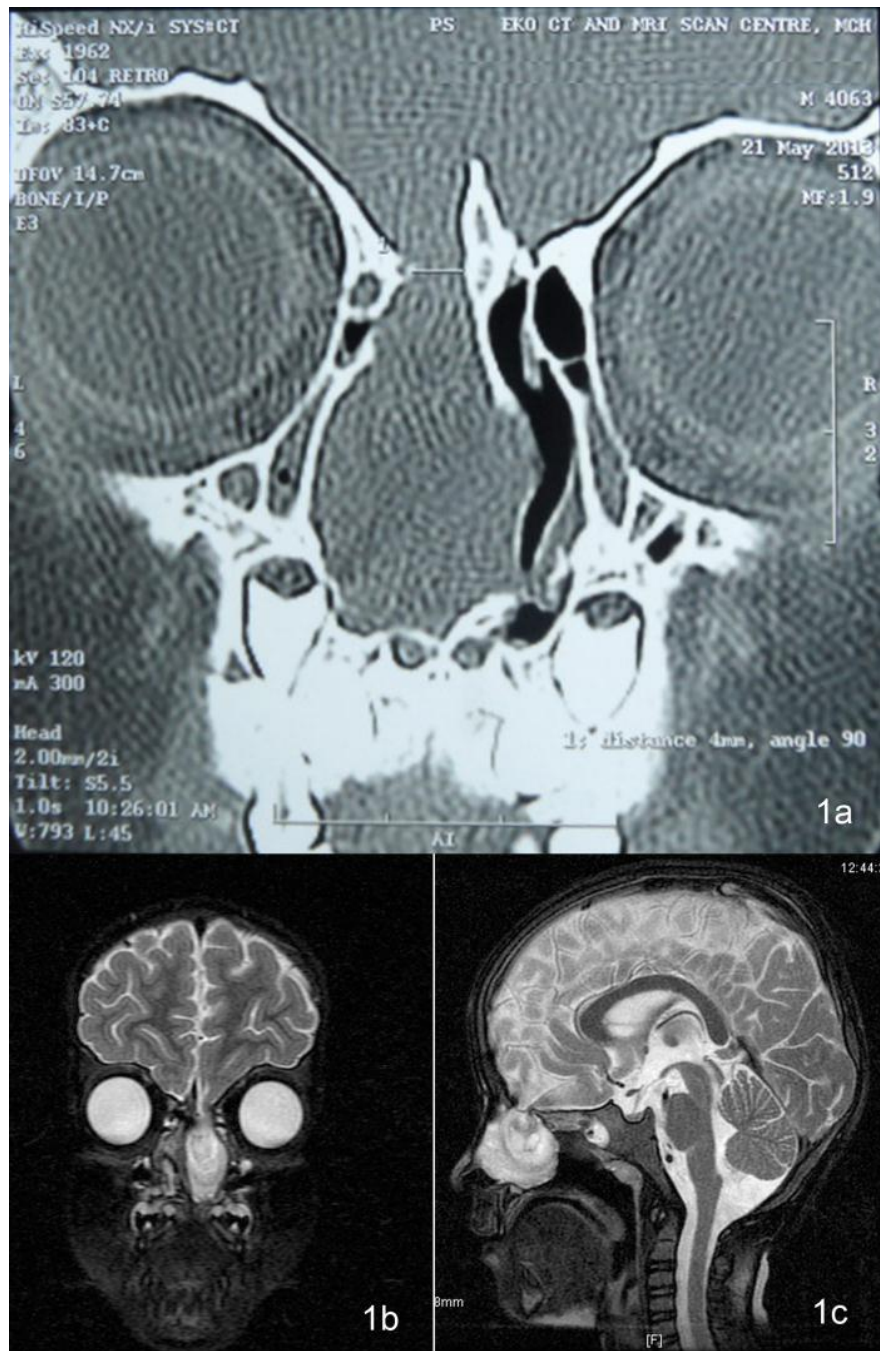

Fig.1: Shows a $4 \mathrm{~mm}$ bony defect in the medial aspect of left cribriform plate (1a) with herniation of mass containing small brain tissue and large CSF $(1 \mathrm{~b} \& \mathrm{c})$ into the nasal cavity suggestive of anterior ethmoidal meningoencephalocele

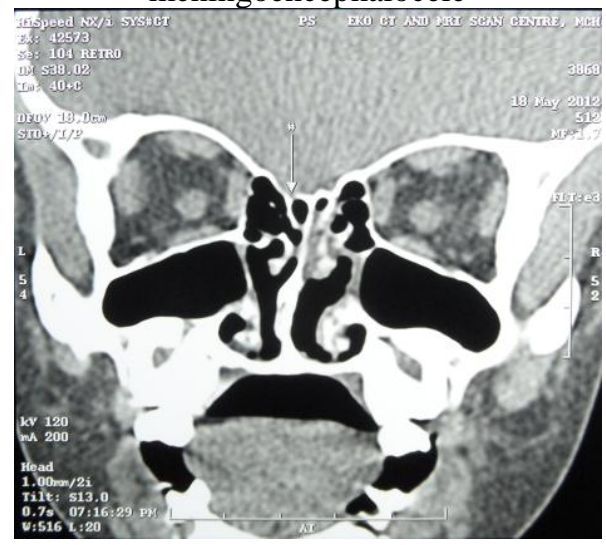

Fig. 2: HRCT scan coronal view shows a bony gap of about $1 \mathrm{~mm}$ (arrow) in the medial part of left cribriform plate but the CSF leak cannot be demonstrated. 


\section{CONCLUSION}

The etiologies of CSF rhinorrhea are varied; include traumatic, non-traumatic and spontaneous. Traumatic leak is seen either following accidental head injury or surgical procedure, accounts for approximately $80 \%$ and $16 \%$ respectively. The non-traumatic leaks are associated with meningocele or meningoencephalocele, congenital skull base defects, high intracranial pressure secondary to erosive diseases, hydrocephalous and brain tumours. Spontaneous CSF leak is idiopathic in origin [3,4].

Certain condition like unilateral autonomic rhinitis or rhinorrhea following the spontaneous rupture of a mucus retention cyst in the maxillary sinus can mimic CSF leak. Therefore it is imperative to establish the diagnosis of CSF rhinorrhea beyond doubt before surgical correction. One may suspect the presence of CSF when the glucose concentration in the fluid sample exceeds $30 \mathrm{mg} / \mathrm{dL}$. Glucose oxidase testing is not acceptable because of its poor predictive value. Test for beta-2 transferrin by immunofixation has been considered the gold standard with a sensitivity and specificity of $100 \%$ and $95 \%$ respectively [1,5].

Once confirmed, accurate localization of the defect is critical in the management of CSF rhinorrhea, particularly if operative management is considered. HRCT scan with both the coronal and axial views have been considered as the initial non-invasive radiological investigation which can detect bony defect in up to $84 \%$ of cases but CSF leak is poorly detected [1]. CT cisternography is considered to be the gold standard with detection rate up to $92 \%$ when the CSF leak is active but with intermittent leak the detection rate drops. In contrast, T2weighted MRI and MR cisternography is a non-invasive method for assessing the presence of intranasal CSF without radiation risk; however spatial resolution is poor and can't detect bony details. The sensitivity of MR cisternography is reported to be $85 \%$ to $92 \%$, with $100 \%$ specificity. To overcome the shortcomings of both HRCT scan and MRI, recently a combination of both the technique have been considered as the as the primary diagnostic method of choice for the patients with CSF rhinorrhea [6,7].

The optimal management decision depends on a variety of factors. Surgery is the mainstay of treatment. Traditionally, the two options were available; intracranial approach via craniotomy and extradural external approach. In spite of associated high mortality and morbidity, this approach is still be the method of choice if there is any co-existing intracranial pathology causing the leak that require resection. In contrast, an extradural extracranial approach remains the method of choice to detect most leaks in the posterior wall of the frontal sinus or defects larger than $5 \mathrm{~cm}$ with success rate vary from 76-100\% [1,2]. Endoscopic closure has revolutionised the surgical management of CSF rhinorrhea and now considered the approach of choice for repairing majority of CSF leaks. It has minimal morbidity with the excellent success rate of 90-97\% [8].

\section{REFERENCES}

[1]. Marshall AH and Jones NS. Cerebrospinal fluid rhinorrhea. In: Gleeson M, Browning GG, Bur-ton MJ, Clarke R, Hibbert J, Jones NS, et al, editors. Scott-Brown's Otorhinolarynglogy, Head and Neck Suregery. 7th edition. London: Hodder Arnold; 2008.pp.1636-44.

[2]. Jones NS, Becker DG. Advances in the management of CSF leaks. BMJ. 2001;322(7279):122-3.

[3]. Singh R, Hazarika P, Nayak DR, Balakrishnan R, Hazarika M, Singh A. Endoscopic repair of cerebrospinal fluid rhinorrhea Manipal experience. Indian J Otolaryngol Head Neck Surg. 2009; 61(1):14-8.

[4]. Tahir MZ, Khan MB, Bashir MU, Akhtar S, Bari E. Cerebrospinal fluid rhinorrhea: An institu-tional perspective from Pakistan. Surg Neurol Int. 2011;2:174.

[5]. Nandapalan V, Watson ID, Swift AC. Beta-2-transferrin and cerebrospinal fluid rhinorrhoea. Clin Otolaryngol 1996; 21(3):259-64

[6]. Mostafa BE, Khafagi A. Combined HRCT and MRI in the detection of CSF rhinorrhea. Skull Base. 2004; 14(3):157-62.

[7]. Prosser JD, Vender JR, Solares CA. Traumatic cerebrospinal fluid leaks. Otolaryngol Clin North Am. 2011;44 (4):857-73.

[8]. Hegazy HM, Carrau RL, Snyderman CH, Kassam A, Zweig J. Transnasal endoscopic repair of cerebrospinal fluid rhinorrhoea: a meta-analysis. Laryngoscope 2000;110 (7):1166-72. 